Article

\title{
Generalized Multiplicative Risk Apportionment
}

\author{
Hongxia Wang \\ College of Economics and Management, Nanjing Forestry University, Nanjing 210037, China; whx2016@njfu.edu.cn \\ Received: 12 April 2019; Accepted: 4 June 2019; Published: 12 June 2019 \\ check for \\ updates

\begin{abstract}
This work examines apportionment of multiplicative risks by considering three dominance orderings: first-degree stochastic dominance, Rothschild and Stiglitz's increase in risk and downside risk increase. We use the relative $n$ th-degree risk aversion measure and decreasing relative $n$ th-degree risk aversion to provide conditions guaranteeing the preference for "harm disaggregation" of multiplicative risks. Further, we relate our conclusions to the preference toward bivariate lotteries, which interpret correlation-aversion, cross-prudence and cross-temperance.
\end{abstract}

Keywords: multiplicative risk apportionment; mean-preserving spreads; downside risk increase

JEL Classification: D81

\section{Introduction}

Since Eeckhoudt and Schlesinger (2006) showed the simple lottery pair characterization for such concepts of risk apportionment as risk prudence and temperance by the idea of "harm disaggregation", plenty of both theoretical and empirical/experimental research has explored preferences over various lottery pairs.

Consider the statistically independent random variables $X_{i}, Y_{i}, i=M, N$. Eeckhoudt et al. (2009a) looked at a preference toward the additive 50-50 lottery $\left[X_{N}+Y_{M}, Y_{N}+X_{M}\right]$ and 50-50 lottery $\left[X_{N}+\right.$ $X_{M}, Y_{N}+Y_{M}$ ] to extend Eeckhoudt and Schlesinger's (Eeckhoudt and Schlesinger 2006) work by defining relatively "good" and "bad" via stochastic dominance. Recently, Ebert et al. (2018) reinterpreted their observation as "mutual aggravation of risk changes". Although Jokung (2011) and Denuit and Rey (2013). among others, developed previous results to the bivariate framework, most researchers focus on additive lottery pairs.

Decision-makers often face multiple risks that are multiplicative. For example ${ }^{1}$, a firm may face a random pre-tax profit and a random tax rate simultaneously; an employee may get a random nominal wage income, while suffer the impact of a price deflator. Thus, we pay attention to the multiplicative counterpart of additive lotteries: $\left[X_{N} Y_{M}, Y_{N} X_{M}\right]$ and $\left[X_{N} X_{M}, Y_{N} Y_{M}\right]$. Eeckhoudt et al. (2009b) and Wang and $\mathrm{Li}$ (2010), among others, use this type of multiplicative lottery pairs to explore "multiplicative risk apportionment", but restrict $X_{M}=1$ and $Y_{M}=1-k(0<k<1)$; Chiu et al. (2012) and Denuit and Rey (2014) also investigated similar lotteries with an additive risk or elementary correlation increasing transformations, but still assumed $X_{M}$ and $Y_{M}$ are different constants.

In this work, the multiplicative 50-50 lotteries allow that $X_{M}$ and $Y_{M}$ take random variables; however, $X_{i}$ also dominates $Y_{i}, i=M, N$, by three well-known dominance orderings: first-degree stochastic

1 For more examples, we refer to, e.g., Franke et al. (2006). 
dominance, Rothschild and Stiglitz's increase in risk (Rothschild and Stiglitz 1970) and downside risk increase (Menezes et al. 1980), which are extensively used to characterize risk changes. ${ }^{2}$ Let $u(x)$ be the utility function of decision-maker, denote as $R R A^{(n)}$ the coefficient of relative $n$ th-degree risk aversion $-x \frac{u^{(n+1)}(x)}{u^{(n)}(x)}{ }^{3}$ We present the relationship between the multiplicative lottery preference ordering with $R R A^{(n)}$ involving the coefficient of relative risk aversion $-x \frac{u^{\prime \prime}(x)}{u^{\prime}(x)}$, the coefficient of relative risk prudence $-x \frac{u^{\prime \prime \prime}(x)}{u^{\prime \prime}(x)}$ and so on. Similar to previous literature on optimal decision-making under risks ${ }^{4}$, our conclusion depends on the comparison between $R R A^{(n)}$ and benchmark value $n$. Moreover, we find it is meaningful that $R R A^{(n)}$ is compared to benchmark value $2 n$. Combining with discussion of benchmark values for $R R A^{(n)}$, under the assumption of decreasing $R R A^{(n)}$, which characterizes the effect of wealth level on the strength of risk aversion ${ }^{5}$, we further provide some sufficient conditions for the multiplicative lottery preference ordering.

Our work proceeds as follows. In the next section, we present our model and explain multiplicative risk apportionment in terms of both "harms disaggregation" (Eeckhoudt et al. 2009b) and "mutual aggravation of risk changes" (Ebert et al. 2018). Section 3 shows the main result. Section 4 applies our model to discuss the concepts for correlation-aversion, cross-prudence and cross-temperance. Section 5 concludes this work.

\section{Model}

We start by reviewing three dominance orderings: first-degree stochastic dominance (FSD), Rothschild and Stiglitz's increase in risk (RSIR) and downside risk increase (DRI). Let $F$ and $G$ be the cumulative distribution functions of two random variables with supports contained in $[a, b]$. $F$ dominates $G$ via FSD if $F(x) \leq G(x)$ for all $x \in[a, b]$, with strict inequality at some $x$, which implies $F$ has a bigger mean than $G .^{6} F$ dominates $G$ via RSIR if $G$ is obtained from $F$ by a sequence of mean-preserving spreads (Rothschild and Stiglitz 1970). Similarly, $F$ dominates $G$ via DRI if $G$ is gotten from $F$ by a sequence of mean-variance-preserving transformations (Menezes et al. 1980). Denote $F^{1}(z)=F(z), F^{k+1}(z)=$ $\int_{a}^{z} F^{k}(y) d y$ and $G^{1}(z)=G(z), G^{k+1}(z)=\int_{a}^{z} G^{k}(y) d y$, where $k=1,2, \cdots$. Ekern (1980) generalized these dominance orderings to $n$ th-degree risk increase, which corresponds to FSD, RSIR, and DRI when $n=1,2,3$, respectively. For convenience, this work also use the term " $n$ th-degree risk increase" to represent these three dominance orderings.

Definition 1. F dominates $G$ via nth-degree risk increase if: (i) $G^{k}(b)=F^{k}(b)$, for $k=1,2, \cdots$; and (ii) $G^{n}(z) \geq$ $F^{n}(z)$, for all $a<z<b$. Moreover, this inequality is strict for some $z$.

Rothschild and Stiglitz (1970) and Menezes et al. (1980), among others, linked these dominance orderings to the sign of derivatives of utility function within the expected-utility framework. Define that decision-maker $u(x)$ is $n$ th-degree risk averse if $(-1)^{n} u^{(n)}(x)<0$ for all $x \in[a, b]$. Ekern (1980) extended their results.

\footnotetext{
See, e.g., Eeckhoudt and Schlesinger (2008) and Liu and Neilson (2018), among others.

Here, $u^{(n)}(x)=\frac{d^{n} u(x)}{d x^{n}}$

For more details, we refer to Eeckhoudt and Schlesinger (2008) and Chiu et al. (2012, p. 160), among others.

For more details, we refer to Franke et al. (2006), Jokung (2013), among others.

See, e.g., Ingersoll (1987).
} 
Lemma 1. F dominates $G$ via nth-degree risk increase if and only if $\int_{a}^{b} u(t) d F \geq \int_{a}^{b} u(t) d G$, for every nth-degree risk aversion decision-maker $u(x)$.

Next, we consider the positive mutually independent random variables $X_{N}, Y_{N}, X_{M}, Y_{M}$, where $X_{i}$ dominates $Y_{i}$ via $i$ th-degree risk increase, $1 \leq i=M, N \leq 3$. Based on additive lottery pairs of Eeckhoudt et al. (2009a), we introduce the following multiplicative 50-50 lotteries:

$$
\begin{aligned}
& A=\left[X_{M} Y_{N}, Y_{M} X_{N}\right] \\
& B=\left[X_{M} X_{N}, Y_{M} Y_{N}\right] .
\end{aligned}
$$

Without loss of generality, we assume $M \leq N$. For $M$ th and $N$ th-degree risk aversion decision-maker, $Y_{i}$ is a "relatively bad" risk, $i=M, N$. Thus, the lottery $A$ means one of both "harms" occurs for certain, whereas the lottery $B$ means both "harms" occur simultaneously or neither occur. That is, following Eeckhoudt and Schlesinger (2006), we can state $A \succeq B$ as the preference for "multiplicative harm disaggregation".

Under the expected-utility framework, $A \succeq B$ is equivalent to

$$
E u\left(X_{M} X_{N}\right)-E u\left(Y_{M} Y_{N}\right) \geq\left[E u\left(X_{M} X_{N}\right)-E u\left(Y_{M} X_{N}\right)\right]+\left[E u\left(X_{M} X_{N}\right)-E u\left(X_{M} Y_{N}\right)\right]
$$

In the above inequality, the left side indicates utility reduction due to both risk changes from $X_{i}$ to $Y_{i}, i=M, N$, simultaneously. The first (second) term in the right side represents utility reduction due to risk change from $X_{M}\left(X_{N}\right)$ to $Y_{M}\left(Y_{N}\right)$. Thus, $A \succeq B$ exhibits a preference for facing risk increases one at a time to suffering two risk increases at once. That is, following Ebert et al. (2018), we can explain $A \succeq B$ by borrowing terminology "mutual aggravation" Kimball (1993) of multiplicative risk changes rather than "harm disaggregation".

\section{Main Result}

Firstly, we have the following conclusions for $A \succeq B$.

Proposition 1. For $N$ th-degree risk aversion decision-maker $u(x)$ :

(i) When $M=1, N=1,2$ or $3, A \succeq B$ if and only if $R R A^{(N+1)} \geq N$.

(ii) When $M=2, N=2$ or $3, A \succeq B$ if and only if

$$
R R A^{(N+1)}\left(2 N-R R A^{(N+2)}\right) \leq N(N-1) .
$$

(iii) When $M=3, N=3, A \succeq B$ if and only if

$$
6-18 R R A^{(4)}+9 R R A^{(4)} R R A^{(5)}-R R A^{(4)} R R A^{(5)} R R A^{(6)} \leq 0 .
$$

Proof. See Appendix A.

Result (i) shows that, when $X_{M}$ dominates $Y_{M}$ via FSD, a decision maker prefers $A$ to $B$ if and only if $R R A^{(N+1)}$ is above the benchmark $N$. Result (ii) indicates that, when $X_{M}$ dominates $Y_{M}$ via RSIR, the values of $R R A^{(N+1)}$ and $R R A^{(N+2)}$ together govern whether $A \succeq B$ holds. ${ }^{7}$ Result (iii) demonstrates

7 For the case of the second-order stochastic dominance, one can further explore by adopting similar approach and noticing the relationship between stochastic dominance and $n$ th-degree risk increase. 
we need to evaluate the values of $R R A^{(4)}, R R A^{(5)}$ and $R R A^{(6)}$ when $X_{M}$ dominates $Y_{M}$ via DRI. That is, Proposition 1 reveals that $R R A^{(N+1)} \geq N$ is insufficient to assure $A \succeq B$ when $M=2,3$. Of course, the equivalent conditions for $M=2,3$ may be a little difficult to be understood. However, we can give simple sufficient conditions, which have been verified in previous literature.

To achieve our goal, we provide some properties of decreasing $R R A^{(n+1)}$. Since $\left(R R A^{(n+1)}\right)^{\prime}=$ $\frac{1}{x} R R A^{(n+1)}\left(1-R R A^{(n+2)}+R R A^{(n+1)}\right)$ for any $x>0$, we have

$$
\left(R R A^{(n+1)}\right)^{\prime} \leq 0 \Leftrightarrow R R A^{(n+2)} \geq R R A^{(n+1)}+1 ;
$$

moreover, if $R R A^{(n+1)} \geq n$, then

$$
\left(R R A^{(n+1)}\right)^{\prime} \leq 0 \Rightarrow R R A^{(n+2)} \geq n+1 .
$$

Following Proposition 1, we have the following corollary.

Corollary 1. For Nth-degree risk aversion decision-maker $u(x)$, when $M=2, N=2$ or 3 , suppose one of the following conditions holds:

(i) $R R A^{(N+1)} \geq N$ and $R R A^{(N+1)}$ is decreasing;

(ii) $R R A^{(N+1)} \leq N$ and $R R A^{(N+2)} \geq N+1$; and

(iii) $R R A^{(N+2)} \geq 2 N$.

Then, $A \succeq B$.

Proof. It is evident to obtain (ii) and (iii) by observing Equation (2). Next, we show (i). When $2 N-$ $R R A^{(N+2)} \leq 0$, by (iii), Equation (2) holds. When $2 N-R R A^{(N+2)} \geq 0$, we complete the proof as follows. By Equations (4) and (5), under $R R A^{(N+1)} \geq N$, decreasing $R R A^{(N+1)}$ means

$$
R R A^{(N+2)} \geq R R A^{(N+1)}+1
$$

and

$$
R R A^{(N+2)} \geq N+1 .
$$

Thus, we have

$$
R R A^{(N+1)}\left(2 N-R R A^{(N+2)}\right) \leq\left(R R A^{(N+2)}-1\right)\left(2 N-R R A^{(N+2)}\right) \leq N(N-1) .
$$

The first inequality in Equation (8) is obtained by Equation (6). The second inequality holds since $\left(R R A^{(N+2)}-1\right)\left(2 N-R R A^{(N+2)}\right)$ has the maximum value $N(N-1)$ at $R R A^{(N+2)}=N+1$ when Equation (7) holds. Then, recalling (ii) in Proposition 1, by Equation (8), we show $A \succeq B$.

Decreasing $R R A^{(n+1)}$ means the increase of wealth level will decrease the degree of risk aversion. Condition (i) in Corollary 1 uses decreasing $R R A^{(N+1)}$ to characterize the sufficient condition for $A \succeq$ $B$. Franke et al. (2006) and Jokung (2013), among others, clarified the role of decreasing $R R A^{(N+1)}$ on multiplicative risk vulnerability. Here, we reveal how decreasing $R R A^{(N+1)}$ guarantees preference for "multiplicative harm disaggregation". For example, when $M=N=2$, by Corollary 1 , if the coefficient of relative risk prudence, which is above 2, is decreasing in the wealth level, then a risk aversion decision-maker exhibits the preference ordering: $A \succeq B$. As a product, we further confirm the exploration about decreasing $R R A^{(N+1)}$ is helpful for analyzing optimal decision-making with multiple multiplicative risks. 
Condition (ii) in Corollary 1 suggests the preference trait may have a reversal as the changes in the dominance ordering between $X_{M}$ and $Y_{M}$. Since, for a decision-maker with $R R A^{(N+1)} \leq N$, when $M=1$, Result (i) in Proposition 1 implies she exhibits a preference for mutual aggravation of multiplicative risks, i.e., $A \preceq B$; however, when $M=2$, if $R R A^{(N+2)} \geq N+1$ holds, then she exhibits a preference for "multiplicative harm disaggregation", i.e., $A \succeq B$. Nevertheless, Condition (ii) still argues $n$ is a kind of benchmark value for $R R A^{(n+1)}$. In fact, in the literature, besides $n, R R A^{(n+1)}$ is also compared to other benchmark values. For example, Gollier (2010) and Denuit and Rey (2014), among others, chose $n-1$ as a benchmark of $R R A^{(n+1)}$. Condition (iii) of Corollary 1 shows $R R A^{(N+2)} \geq 2 N$ alone is sufficient to guarantee $A \succeq B$, which proposes another larger benchmark value $2 N$ for $R R A^{(N+1)}$ noting that $2 N \geq N+1$. By (i) in Proposition 1 , we know $R R A^{(N+2)} \geq 2 N \Rightarrow A \succeq B$ when $M=1$. This means a strong enough preference for $A \succeq B$ with $M=1$ is sufficient to assure $A \succeq B$ with $M=2$.

There are similar sufficient conditions for the case of $M=3$ and $N=3$.

Corollary 2. For prudent decision-maker $u(x)$ (i.e., $u^{\prime \prime \prime}>0$ ), when $M=3$ and $N=3$, suppose one of the following conditions holds:

(i) $R R A^{(4)} \geq 3, R R A^{(5)} \geq 4$ and decreasing $R R A^{(5)}$;

(ii) $R R A^{(4)} \geq 3, R R A^{(5)} \leq 4$ and $R R A^{(6)} \geq 5$; or

(iii) $R R A^{(4)} \geq 3$ and $R R A^{(6)} \geq 9$.

Then, $A \succeq B$.

Proof. The proof is similar to Corollary 1. Please see Appendix A.

To exhibit the economic interpretation of our results, we place emphasis on three dominance orderings: FSD, RSIR, and DRI. ${ }^{8}$ For the case of higher-degree risk increase, one can get similar conditions to Proposition 1, and (ii) and (iii) in Corollaries 1 and 2 by a little complex mathematical derivation; however, we realize that it is not straightforward to generalize (i) in Corollaries 1 and 2 by the method of proofs. Some additional conditions should be introduced, since, for example, as shown in the Appendix, the second inequality in Equation (A5) does not hold when $M=3, N=4$.

\section{Applications}

\subsection{Relate to Multiplicative Risk Apportionment}

The lottery preference on our multiplicative 50-50 lotteries extends the concept of "Multiplicative risk apportionment" by Eeckhoudt et al. (2009b) and Wang and Li (2010). They introduced the following special lotteries:

$$
\begin{aligned}
& A_{n+1}^{*}=\left[x(1-r)\left(1+A_{n}\right) ; x\left(1+B_{n}\right)\right], \\
& B_{n+1}^{*}=\left[x(1-r)\left(1+B_{n}\right) ; x\left(1+A_{n}\right)\right] .
\end{aligned}
$$

where, following from Eeckhoudt and Schlesinger (2006), $B_{1}=B_{2}=[0], A_{1}=[-k]$ and $A_{2}=\left[\tilde{\epsilon}_{1}\right]$; for $n \geq 3, A_{n}=\left[0+B_{n-2} ; \tilde{\epsilon}_{I n t(n / 2)}+A_{n-2}\right], B_{n}=\left[0+A_{n-2} ; \tilde{\epsilon}_{I n t(n / 2)}+B_{n-2}\right] .{ }^{9}$

Now, let $\widetilde{y}=1+A_{n}, \widetilde{x}=1+B_{n}, 0<1-r=k<1, w=x$. then

$$
A_{n+1}^{*}=\left[x(1-r)\left(1+A_{n}\right) ; x\left(1+B_{n}\right)\right],
$$

8 Eeckhoudt and Schlesinger (2008, p. 1331), among others, argued there is little empirical literature considering the case of $n$th degree risk increase, $n \geq 4$.

$9 \operatorname{Int}(y)$ denotes the greatest-integer function, i.e., the greatest integer not exceeding the real number $y$. 


$$
B_{n+1}^{*}=\left[x(1-r)\left(1+B_{n}\right) ; x\left(1+A_{n}\right)\right] .
$$

Obviously, according to Eeckhoudt and Schlesinger (2006), $1+A_{n}$ has more $n$ th-degree risk than $1+B_{n}$ and $x$ FSD $x(1-r)$. Thus, the preference between $A_{n+1}^{*}$ and $B_{n+1}^{*}$ is a special case of our conclusion. The result of Eeckhoudt et al. (2009b) and Wang and Li (2010) is relatively intuitive and simple. However, the characterization is less simple for higher orders. This paper provides a way to characterize generalized multiplicative risk apportionment via lottery pairs.

\subsection{Relate to Preferences over Bivariate Lottery Pairs}

We now adopt the bivariate utility function ${ }^{10} v(x, y)=u(x y)\left(u^{\prime}>0, u^{\prime \prime}<0\right)$ to investigate the work of Eeckhoudt et al. (2007). Assume zero-mean risks: $\tilde{\epsilon}$ and $\tilde{\delta}$ are statistically independent. Let $\operatorname{supp}[x+$ $\tilde{\epsilon}]$ denote the support of $x+\tilde{\epsilon}$. Similar notation also applies to $y+\tilde{\delta}$. When the two variables are multiplicative, following Eeckhoudt et al. (2007), we know that cross-temperance is equivalent to $[(x+$ $\tilde{\epsilon}) y ; x(y+\tilde{\delta})] \succ[x y ;(x+\tilde{\epsilon})(y+\tilde{\delta})]$. Since $x+\tilde{\epsilon}$ and $y+\tilde{\delta}$ dominate $x, y$, respectively, via RSIR, by Corollary 1 , we have the following corollary.

Corollary 3. Suppose one of the three conditions $(N=2)$ in Corollary 1 holds. Then, a decision-maker with $u(x, y)=u(x y)$ is cross-temperate, i.e., exhibits $[(x+\tilde{\epsilon}) y ; x(y+\tilde{\delta})] \succeq[x y ;(x+\tilde{\epsilon})(y+\tilde{\delta})]$ for any $x>0, y>0$, $\operatorname{supp}[x+\tilde{\epsilon}]>0, \operatorname{supp}[y+\tilde{\delta}]>0$.

Similarly, by Proposition 1, we have the following conclusion which covers the lottery pairs interpretation about the coefficients of relative risk aversion and relative prudence in Eeckhoudt et al. (2009b), Wang and Li (2010).

Corollary 4. A decision-maker with $u(x, y)=u(x y)$ is correlation-averse, i.e., exhibits $[(x-k) y ; x(y-c)] \succeq$ $[x y ;(x-k)(y-c)]$ for any $x>0, y>0, x-k>0, y-c>0$, if and only if $R R A^{(2)} \geq 1$.

A decision-maker with $u(x, y)=u(x y)$ is cross-prudent, i.e., exhibits $[(x+\tilde{\epsilon}) y ; x(y-c)] \succeq[x y ;(x+$ $\tilde{\epsilon})(y-c)]$ for any $x>0, y>0, y-c>0, \operatorname{supp}[x+\tilde{\epsilon}]>0$ or $[x(y+\tilde{\delta}) ;(x-k) y)] \succeq[x y ;(x-k)(y+\tilde{\delta}))]$ for any $x>0, y>0, x-k>0, \operatorname{supp}[y+\tilde{\delta}]>0$, if and only if $R R A^{(3)} \geq 2$.

As discussed by Eeckhoudt et al. (2007), the economic and financial decisions under risks often involve many attributes. Our conclusions can be used to examine intertemporal consumption choices with two multiplicative arguments. Thus, our work also extends the interpretations of Eeckhoudt et al. (2007) on the signs of cross-derivatives of utility functions.

\section{Conclusions}

This work is devoted to providing conditions for preferences over multiplicative additive lottery pairs. We extend previous results on multiplicative risk apportionment by restricting our work on the three dominance orders: first-degree stochastic dominance, Rothschild and Stiglitz's increase in risk and downside risk increase. Surprisingly, we find decreasing relative $n$ th-degree risk aversion can be used to analyze preferences for "multiplicative harm disaggregation". This extension is helpful to motivate further study on multiplicative risk apportionment.

Funding: This research was funded by MOE (Ministry of Education in China) Project of Humanities and Social Sciences with Grant Number 19YJC790125. 
Acknowledgments: The author would like to thank the editor and anonymous referees for providing valuable suggestions which have led to significant improvement of this article.

Conflicts of Interest: The author declares no conflict of interest.

\section{Appendix A}

\section{Appendix A.1. Proof of Proposition 1}

Proof. We know

$$
\begin{aligned}
& A \succeq B \\
\Leftrightarrow & \frac{1}{2} E u\left(X_{N} Y_{M}\right)+\frac{1}{2} E u\left(Y_{N} X_{M}\right) \geq \frac{1}{2} E u\left(X_{N} X_{M}\right)+\frac{1}{2} E u\left(Y_{N} Y_{M}\right) \\
\Leftrightarrow & E u\left(X_{N} Y_{M}\right)-E u\left(X_{N} X_{M}\right) \geq E u\left(Y_{N} Y_{M}\right)-E u\left(Y_{N} X_{M}\right) \\
\Leftrightarrow & \left(E u\left(x Y_{M}\right)-E u\left(x X_{M}\right)\right)^{(N)}(-1)^{N} \leq 0 \quad(\text { by Lemma 1) } \\
\Leftrightarrow & E Y_{M}^{N} u^{(N)}\left(x Y_{M}\right)(-1)^{N} \leq E X_{M}^{N} u^{(N)}\left(x X_{M}\right)(-1)^{N} \\
\Leftrightarrow & \frac{d^{M} y^{N} u^{(N)}(x y)(-1)^{N}}{d y^{M}}(-1)^{M} \leq 0 \quad(\text { by Lemma 1). }
\end{aligned}
$$

Thus, when $M=1$,

$$
\begin{aligned}
& A \succeq B \\
\Leftrightarrow & \left.-y x \frac{u^{(N+1)}(x y)}{u^{(N)}(x y)} \geq N \quad \text { (note that }(-1)^{N} u^{(N)}(\cdot)<0\right) \\
\Leftrightarrow & R R A^{(N+1)} \geq N .
\end{aligned}
$$

When $M=2$,

$$
\begin{aligned}
& A \succeq B \\
\Leftrightarrow & N(N-1) y^{N-2} u^{(N)}(x y)(-1)^{N}+2 N y^{N-1} x u^{(N+1)}(x y)(-1)^{N}+y^{N} x^{2} u^{(N+2)}(x y)(-1)^{N} \leq 0 \\
\Leftrightarrow & \left.N(N-1)+2 N x y \frac{u^{(N+1)}(x y)}{u^{(N)}(x y)}+y^{2} x^{2} \frac{u^{(N+2)}(x y)}{u^{(N)}(x y)} \geq 0 \quad \text { note that }(-1)^{N} u^{(N)}(\cdot)<0\right) \\
\Leftrightarrow & N(N-1)-2 N R R A^{(N+1)}+R R A^{(N+1)} R R A^{(N+2)} \geq 0 \\
\Leftrightarrow & R R A^{(N+1)}\left(2 N-R R A^{(N+2)}\right) \leq N(N-1) .
\end{aligned}
$$

When $M=3$,

$$
\begin{aligned}
& A \succeq B \\
& \Leftrightarrow \quad N(N-1)(N-2) y^{N-3} u^{(N)}(x y)(-1)^{N}+N(N-1) x y^{N-2} u^{(N+1)}(x y)(-1)^{N} \\
&+2 N(N-1) y^{N-2} x u^{(N+1)}(x y)(-1)^{N}+2 N y^{N-1} x^{2} u^{(N+2)}(x y)(-1)^{N} \\
&+N y^{N-1} x^{2} u^{(N+2)}(x y)(-1)^{N}+y^{N} x^{3} u^{(N+3)}(x y)(-1)^{N} \geq 0 \\
& \Leftrightarrow \quad N(N-1)(N-2)-3 N(N-1) R R A^{(N+1)}+3 N R R A^{(N+1)} R R A^{(N+2)} \\
& \quad\left.-R R A^{(N+1)} R R A^{(N+2)} R R A^{(N+3)} \leq 0 \quad \text { (note that }(-1)^{N} u^{(N)}(\cdot)<0\right) .
\end{aligned}
$$

Then, let $M=N=3$, by Equation (A4), we get (iii). 
Appendix A.2. Proof of Corollary 2

Proof. Recalling Equation (A4), when $R R A^{(N+1)} \geq N$, it is easy to find Equation (A4) can be guaranteed by

$$
\begin{aligned}
& -3 N(N-1)+3 N R R A^{(N+2)}-R R A^{(N+2)} R R A^{(N+3)} \leq-(N-1)(N-2) \\
\Leftrightarrow \quad & \left.R R A^{(N+2)}\left(3 N-R R A^{(N+3)}\right) \leq 2\left(N^{2}-1\right)\right) .
\end{aligned}
$$

where we only show the proof of (i) when $3 N-R R A^{(N+3)} \geq 0$. By Equations (4) and (5), under $R R A^{(N+2)} \geq N+1$, decreasing $R R A^{(N+2)}$ implies $R R A^{(N+3)} \geq R R A^{(N+2)}+1$ and $R R A^{(N+3)} \geq N+2$. Thus, we have

$$
R R A^{(N+2)}\left(3 N-R R A^{(N+3)}\right) \leq\left(R R A^{(N+3)}-1\right)\left(3 N-R R A^{(N+3)}\right) .
$$

Moreover, noting $R R A^{(N+3)} \geq N+2$, we know if

$$
\frac{3 N+1}{2} \leq N+2
$$

i.e., $N \leq 3$, then $\left(R R A^{(N+3)}-1\right)\left(3 N-R R A^{(N+3)}\right)$ takes the maximum value $\left.2\left(N^{2}-1\right)\right)$. Then, Equation (A5) holds when $N=3$. Thus, we show (i) is sufficient for $A \succeq B$.

\section{References}

Chiu, W. Henry, Louis Eeckhoudt, Beatrice Rey. 2012. On relative and partial risk attitudes: Theory and implications. Economy Theory 50: 151-67. [CrossRef]

Denuit, Michel, and Béatrice Rey. 2013. Another look at risk apportionment. Journal of Mathematical Economics 49: 335-43. [CrossRef]

Denuit, Michel, and Béatrice Rey. 2014. Benchmark values for higher order coefficients of relative risk aversion. Theory Decision 76: 81-94. [CrossRef]

Ebert, Sebastian, Diego C. Nocetti, and Harris Schlesinger. 2018. Greater Mutual Aggravation. Management Science 64: 2473-972. [CrossRef]

Eeckhoudt, L., and H. Schlesinger. 2008. Changes in risk and the demand for saving. Journal of Monetary Economics 55: 1329-336. [CrossRef]

Eeckhoudt, L., H. Schlesinger, and I. Tsetlin. 2009a. Apportioning of risks via stochastic dominance. Journal of Economic Theory 144: 994-1003. [CrossRef]

Eeckhoudt, Louis, and Harris Schlesinger. 2006. Putting risk in its proper place. American Economic Review 96: 280-89. [CrossRef]

Eeckhoudt, Louis, Béatrice Rey, and Harris Schlesinger. 2007. A Good Sign for Multivariate Risk Taking. Management Science 53: 117-24. [CrossRef]

Eeckhoudt, Louis, Johanna Etner, and Fred Schroyen. 2009b. The values of relative risk aversion and prudence: A context-free interpretation. Mathematical Social Sciences 58: 1-7. [CrossRef]

Ekern, Steinar. 1980. Increasing Nth degree risk. Economy Letter 6: 329-33. [CrossRef]

Franke, Günter, Harris Schlesinger, and Richard C. Stapleton. 2006. Multiplicative Background Risk. Management Science 52: 146-53. [CrossRef]

Gollier, Christian. 2010. Discounting, Inequalities and Economic Convergence. Toulouse: Mimeo, Toulouse School of Economics.

Ingersoll, Jonathan E. 1987. Theory of Financial Decision Making. Totowa: Rowman and Littlefield.

Jokung, Octave. 2011. Risk apportionment via bivariate stochastic dominance. Journal of Mathematical Economics 47: 448-52. [CrossRef] 
Jokung, Octave. 2013. Changes in multiplicative background risk and risk-taking behavior. Theory Decision 74: $127-49$. [CrossRef]

Kimball, Miles S. 1993. Standard risk aversion. Econometrica 61: 589-611. [CrossRef]

Liu, Liqun, and William S. Neilson. 2018. Alternative Approaches to Comparative nth-Degree Risk Aversion. Management Science, forthcoming. [CrossRef]

Menezes, C. F., C. Geiss, and John Tressler. 1980. Increasing downside risk. American Economic Review 70: 921-32.

Rothschild, Michael, and Joseph Stiglitz. 1970. Increasing risk I: A definition. Journal of Economic Theory 2: $225-43$. [CrossRef]

Wang, Jianli, and Jingyuan Li. 2010. Multiplicative risk apportionment. Mathematical Social Sciences 60: 79-81. [CrossRef]

(C) 2019 by the author. Licensee MDPI, Basel, Switzerland. This article is an open access article distributed under the terms and conditions of the Creative Commons Attribution (CC BY) license (http:/ / creativecommons.org/licenses/by/4.0/). 